\title{
Locked-In Syndrome
}

National Institute of Neurological Disorders and Stroke (NINDS)

\section{Source}

National Institute of Neurological Disorders and Stroke (NINDS). Locked-In Syndrome

Information Page.

Locked-in syndrome is a rare neurological disorder characterized by complete paralysis

of voluntary muscles in all parts of the body except for those that control eye

movement. It may result from traumatic brain injury, diseases of the circulatory system,

diseases that destroy the myelin sheath surrounding nerve cells, or medication overdose.

Individuals with locked-in syndrome are conscious and can think and reason, but are

unable to speak or move. The disorder leaves individuals completely mute and paralyzed.

Communication may be possible with blinking eye movements 